\section{'Blues' music and psychiatry}

DEAR SIRS

I was interested to read Dr Jelley's article on the relationship between 'Blues' music and psychiatry (Psychiatric Bulletin, April 1990, 14, 227-229). Dr Jelley appears to study the relationship in terms of content and does not pay much attention to the form or style of the music. His central thesis concerns the content or words of Blues songs and he suggests that the singer and listener benefit in a psychotherapeutic way from this communication. He does however briefly mention that 'sad' music has an effect in reducing anxiety.

The key feature of Blues music is its form, including a simple 12 bar structure and use of particular scales, a major and minor pentatonic scale and bent notes (Shaar Murray, 1989). Music based on these scales, as in 'sad' music based on the standard minor scales, can be quite beautiful and evocative of emotions. From its origins in the USA at the turn of the century, Blues music has spread and been adapted in various ways, e.g. to form rhythm and blues music. Its influence on popular music can be heard from Elvis Presley and the Beatles to modern day pop music and it appears to have become a musical norm for western popular music very much like other scales and forms have become standards in other times and cultures. Blues music has also been closely linked to the guitar, especially the electric guitar.

Thus although the words of Blues songs may be important, I would suggest that the musical form and its expression on a particular instrument by an individual are equally of significance and perhaps it is the interplay between these various factors that gives Blues music such a wide appeal.

The North Wales Hospital

Denbigh, Clwyd LL15 5SS

JEFF JONES

\section{Reference}

ShaAr Murray, C. (1989) Crosstown Traffic. Jimi Hendrix and Post-War Pop. London: Faber \& Faber, pp. 129-152.

\section{Use of mental hospital sites}

\section{DeAR SirS}

I was interested to read the article on "The Future of Mental Hospital Sites" (Psychiatric Bulletin, April 1990, 14, 245-248).

One of the proposed uses of these sites was the provision of Industrial Therapy Units. In the current climate of high unemployment might it not be more appropriate to provide centres for social and occupational therapies rather than industrial therapy units? I have recently worked in an industrial therapy centre which has had to change its direction as facilities for sheltered employment and possibilities of employment for those with chronic mental illness become less likely. It could be argued that employment opportunities for the mentally ill should be improved and perhaps positive discrimination should be encouraged. However, I feel we should be examining day care facilities and industrial rehabilitation in view of the current situation.

\section{Rubery Hill Hospital \\ Birmingham B45 9BB}

G. MILNER

\section{New long-stay patients}

\section{DEAR SIRS}

I read with interest the report by Todd and his colleagues about new long-stay male schizophrenic patients (Psychiatric Bulletin, April 1990, 14, 210 212). I have recently carried out a survey of all new long-stay patients (those resident for more than one year) in the Downshire Hospital, a psychiatric hospital with a catchment population of 300,000 , and approximately 1,000 admissions annually.

The survey carried out on 1 November 1989 identified six patients who had remained continuously resident since they had been admitted between 1 April 1987 and 31 October 1988. The annual rate was, therefore, four per year (a figure not dissimilar to Todd's, but one which includes all diagnoses, not just male schizophrenics). None was currently married and all had lived alone or with relatives unable to care for the patient. The population was older than Todd's (mean age of 46 ) with a mean age of 56.5 , only one patient being younger than 50. Similar to Todd's group, the average duration of illness was 20 years, with a range from 2 to 40 years.

Of the six patients, it was surprising to note that only one was schizophrenic; in addition to his psychosis, he was a special care patient. Three $(50 \%)$ had suffered brain damage, one suffered from a bipolar illness and the sixth patient had a depressive illness superimposed on an inadequate personality. Five of the cohort had several failed attempts at rehabilitation and/or resettlement.

There is currently no unit for the adult brain damaged in $\mathbf{N}$. Ireland. As a result, continuing care psychiatric beds tend to be used by these unfortunate patients. Whether these people are placed in the environment most suitable for their needs is open to question. Whatever the answer, if one assumes that the population will live, on average, for a further 1520 years, for the Downshire Hospital's catchment area population of $300,000,60-80$ continuing care beds will be required. 